\title{
Patienten mit einer wahnhaften Depression in der Maßregel nach $\S 63 \mathrm{StGB}$
}

\author{
Georg Stolpmann · Peter Fromberger · Kirsten Jordan • \\ Johannes Schwerdtner $\cdot$ Jürgen L. Müller
}

Eingegangen: 10. April 2010 / Angenommen: 7. Mai 2010 / Online publiziert: 6. Juli 2010

(C) Die Autor(en) 2010. Dieser Artikel ist auf Springerlink.com mit Open Access verfügbar.

\begin{abstract}
Zusammenfassung Die Symptomatik depressiver Erkrankungen wird unter kriminologischen Aspekten oft als protektiv bezeichnet. Bei wahnhafter Depression können aber schwerwiegende Gewalttaten wie erweiterte Suizide verübt werden. Unterbringungsmaßnahmen nach $\S 63$ des Strafgesetzbuches (StGB) werden bei Depressionen selten angeordnet, und wegen der relativ guten Behandlungsprognose ist eine längerfristige stationäre Unterbringung häufig nicht erforderlich. Bei gravierenden Delikten und therapieresistenter Wahnsymptomatik wird eine Maßregelunterbringung dagegen nicht zu umgehen sein. Anhand zweier Kasuistiken wahnhaft bedingter Tötungsdelikte bei depressiver Erkrankung wird auf Fragen der Schuldfähigkeitsbegutachtung und den Begriff des erweiterten Suizids eingegangen.
\end{abstract}

Schlüsselwörter Wahnhafte Depression · Erweiterter Suizid · Schuldfähigkeitsbegutachtung $\cdot$ Maßregel

Dr. G. Stolpmann $(\bowtie) \cdot$ Dipl. Psych. P. Fromberger

Dr. K. Jordan · Prof. Dr. J. L. Müller

Forensische Psychiatrie und Psychotherapie,

Klinik und Poliklinik für Psychiatrie und Psychotherapie,

Georg-August-Universität Göttingen, Rosdorfer Weg 70,

37081 Göttingen, Deutschland

E-Mail: gstolpmann@asklepios.com

Dr. J. Schwerdtner

Fachklinik für Forensische Psychiatrie und Psychotherapie,

Bezirkskrankenhaus, Universitätsstr. 84,

93053 Regensburg, Deutschland

\section{Patients with delusional depression in forensic psychiatric hospitals}

\begin{abstract}
Delinquency of depressed patients numerically plays a minor role in criminal or civil law. Compared with the high prevalence of affective disorders in the general population, the frequency of patients in forensic psychiatric hospitals with the respective disorder as a main diagnosis is low. The spectrum of possible crimes committed by depressed subjects essentially comprises disease-characteristic offences, amongst which cases of extended suicide or attempted suicide are the foremost and most tragic offences. Based on 2 case reports notions of extended suicide as well as questions of forensic assessment are discussed.
\end{abstract}

Keywords Delusional depression - Extended suicide · Forensic assessment $\cdot$ Legal detention

\section{Einleitung}

Depressionen gehören zu den häufigsten psychischen Erkrankungen. Bezogen auf die erwachsene Durchschnittsbevölkerung (Alter 18 bis 65 Jahre) wird für die europäischen Länder eine Zwölfmonatsprävalenz der Major Depression von 6,9\% angegeben [1]. Das angenäherte Lebenszeitrisiko, an einer Depression zu erkranken, wird aufgrund der „Lifetime“-Daten konservativ auf mindestens $14 \%$ geschätzt. Für die BRD bedeutet dies, dass ca. 5-6 Mio. Bundesbürger/Jahr von einer Depression betroffen sind (Tab. 1).

Unter den Diagnosen der im Maßregelvollzug nach $\S 63$ des Strafgesetzbuches (StGB) untergebrachten Patienten spielen Depressionen dagegen nur eine untergeordnete Rolle. Verlässliche Angaben zur Häufigkeit sind nicht zu 
Tab. 1 Jahresprävalenz depressiver Störungen in der Bundesrepublik Deutschland. (Zit. nach [1])
${ }^{a}$ Major Depression (einzelne Episode oder wiederkehrend) und/oder dysthyme Störung.

\begin{tabular}{lcclll} 
& $\begin{array}{l}\text { Irgendeine } \\
\text { depressive } \\
\text { Störung }^{\text {a }}\end{array}$ & $\begin{array}{l}\text { Major } \\
\text { Depression (MD) }\end{array}$ & $\begin{array}{l}\text { MD, einzelne } \\
\text { Episode }\end{array}$ & $\begin{array}{l}\text { MD, } \\
\text { wiederkehrend }\end{array}$ & $\begin{array}{l}\text { Dysthyme } \\
\text { Störung }\end{array}$ \\
\hline Gesamt & 10,9 & 8,3 & 4,3 & 4,0 & 4,5 \\
$18-29$ & 9,5 & 8,0 & 4,7 & 3,4 & 2,7 \\
$30-39$ & 9,7 & 7,5 & 3,1 & 4,4 & 3,8 \\
$40-49$ & 12,4 & 9,8 & 5,4 & 4,4 & 5,3 \\
$50-65$ & 11,6 & 8,1 & 4,2 & 3,9 & 5,7 \\
Frauen & 14,2 & 11,2 & 5,1 & 6,1 & 5,8 \\
$18-29$ & 11,5 & 9,5 & 4,6 & 4,9 & 3,5 \\
$30-39$ & 12,4 & 10,0 & 3,5 & 6,5 & 4,7 \\
$40-49$ & 16,6 & 14,0 & 7,2 & 6,9 & 6,4 \\
$50-65$ & 15,6 & 11,3 & 5,4 & 5,9 & 7,6 \\
Männer & 7,6 & 5,5 & 3,4 & 2,0 & 3,2 \\
$18-29$ & 7,5 & 6,6 & 4,8 & 1,9 & 1,8 \\
$30-39$ & 7,2 & 5,1 & 2,8 & 2,3 & 3,0 \\
$40-49$ & 8,3 & 5,7 & 3,7 & 2,0 & 4,1 \\
$50-65$ & 7,4 & 4,8 & 2,9 & 1,9 & 3,8 \\
\hline
\end{tabular}

finden, zumal in den Statistiken affektive Psychosen nicht differenziert werden. Leygraf [2] nennt einen Anteil von $1,2 \%$ affektiver Psychosen bei den Einweisungsdiagnosen und Seifert u. Leygraf [3] dagegen 0,4\%.

Von einigen Autoren wird die depressive Symptomatik (Gehemmtheit, Skrupelhaftigkeit und Selbstentwertungstendenzen der Depressionskranken, ihre gewissenhaften bis ethisch-rigiden Züge im Sinne des „Typus melancholicus“ [4]) als Schutz vor delinquentem Handeln bezeichnet [5, 6]. Allerdings besteht eine besondere Deliktgefährdung bei schweren Depressionen. So kann etwa ein depressiver Verarmungswahn in delinquentem Verhalten in Form von Diebstahl, Banküberfall, Versicherungsbetrug o. Ä. münden, und im Rahmen suizidaler Handlungen kommen Gewalttaten bei schweren Depressionen vor. Bei erheblichen Straftaten kann eine Depression zur Maßregelunterbringung führen, wenn die Gefährlichkeitsprognose ungünstig ausfällt. Die prognostische Einschätzung des Risikopotenzials einer depressiven Erkrankung erfordert es, neben der speziellen Depressionssymptomatik insbesondere die Fragen der Behandelbarkeit zu berücksichtigen. Bei wahnhaften Depressionen ist eine ambulante Behandlung mit deutlich geringeren Erfolgsaussichten verknüpft als bei einer erstmanifesten Depression ohne psychotische Symptome.

\section{Symptome der Major Depression (nach DSM-IV ${ }^{1}$ )}

Hauptsymptome der Depression sind eine gedrückte Stimmung, Interessenverlust, allgemeine Freudlosigkeit und eine Verminderung des Antriebs. Es lassen sich vielfach eine verminderte Konzentration und Aufmerksamkeit, ein

\footnotetext{
${ }^{1}$ Diagnostic and Statistical Manual of Mental Disorders IV.
}

eingeschränktes Selbstwertgefühl und reduziertes Selbstvertrauen, Schlaf- und Appetitstörungen sowie Suizidgedanken und auch Suizidhandlungen feststellen. Bei einem gewissen Anteil der depressiven Patienten treten mehr oder weniger ausgeprägte Wahnsymptome auf. Häufigkeitsangaben wahnhaften Erlebens bei Depression schwanken in Abhängigkeit von der betrachteten Gruppe zwischen 10 und $45 \%$. Wolfersdorf et al. [7] ermittelten 8-12\% Depressive mit Wahnsymptomatik unter 3392 kumulativen Aufnahmen einer psychiatrischen Klinik im Zeitraum von 1977 bis 1994. In der Studie von Maj et al. [8] fanden sich bei fast $25 \%$ der hospitalisierten Patienten $(\mathrm{n}=61)$ mit einer Major-Depression-Diagnose und bei fast $15 \%$ der ambulanten Patienten $(\mathrm{n}=391)$ Wahnsymptome in der Indexepisode.

Die häufigsten Wahnthemen einer schweren psychotischen Depression sind Versündigungs-/Verschuldungswahn, Verarmungswahn, hypochondrischer Wahn und nihilistischer Wahn [5]. Bei der wahnhaften Depression ist die depressive Herabgestimmtheit gleichzeitig mit der Wahnsymptomatik vorhanden, d. h. affektive und kognitive Störungen treten zeitgleich auf. Es können akustische Halluzinationen vorkommen, die dem Betroffenen sein Versagen, seine Schuldhaftigkeit sowie Wertlosigkeit in Form von Stimmen vorhalten und ihn ggf. zum Suizid auffordern (Tab. 2).

Die Wahnsymptome können stimmungskongruent sein, d. h. Wahninhalt oder Halluzinationen stimmen mit depressiven Themen überein. Es kann aber auch stimmungsinkongruente Merkmale geben, die keinen unmittelbaren Bezug $\mathrm{zu}$ den depressiven Themen haben (z. B. Gedankenausbreitung, -eingebung, Kontrollwahn, Verfolgungswahn). In der Studie von Maj et al. [8] hatten 85 ambulante Patienten $(12,8 \%)$ nur synthyme Wahnsymptome (,mood-congruent delusions"), 13 Patienten (2,9\%) hatten nur parathyme 
Tab. 2 Häufigkeit der Wahnthemen [5]

Prozentzahlen; die Zahlen in Klammern sind Prozente, bezogen auf 23 depressive Patienten mit Wahn.

\begin{tabular}{|c|c|c|c|c|}
\hline & Prospektive & \multicolumn{3}{|c|}{ Retrospektive Untersuchung } \\
\hline & Kuhs 1990/1991 & \multicolumn{3}{|l|}{ Tölle u. Wefelmeyer 1987} \\
\hline & $\mathrm{n}=160$ & \multicolumn{3}{|l|}{$\mathrm{n}=421$} \\
\hline & & Wahn im engeren Sinn & Vorstadien & Gesamt \\
\hline Depressive Patienten mit Wahn & 14,4 & 20,9 & 22,6 & 43,5 \\
\hline Themen des Wahns & & & & \\
\hline - Schuldwahn & $11,9(82,6)$ & 12,6 & 19,2 & 31,8 \\
\hline - Armutswahn & $7,5(52,1)$ & 5,0 & 19,0 & 24,0 \\
\hline - Krankheitswahn & $5,6(38,9)$ & 3,1 & 5,7 & 8,8 \\
\hline - Andere Wahnthemen & $6,3(43,8)$ & 12,1 & 6,2 & 18,3 \\
\hline
\end{tabular}

Wahnsymptome (,mood-incongruent delusions“), und 8 Patienten $(1,8 \%)$ hatten synthyme sowie parathyme Wahnsymptome. Bezüglich demografischer, historischer und Variablen der Ersterkrankung unterschieden sich Patienten mit stimmungskongruentem Wahn nicht von denen mit einem stimmungsinkongruenten Wahn.

Eine Major Depression mit wahnhafter Symptomatik ist forensisch dem ersten Eingangskriterium (krankhafte seelische Störung) des $§ 20 \mathrm{StGB}$ zu subsumieren.

Fallbericht 1 Herr B. war zum Tatzeitpunkt (November 2007) 59 Jahre alt, verheiratet und seit ca. 6 Monaten wegen „Rückenschmerzen“ und „Nervosität" berentet. Seit 2 Jahren befand er sich wegen Depression in Behandlung und war 3 Wochen vor der Tat mit der Diagnose chronifizierte depressive Problematik aus stationärer Behandlung entlassen worden. Er wurde ambulant mit Antidepressiva und Neuroleptika weiterbehandelt.

Bei einem Verkehrsunfall 12 Wochen vor der Tat war die Ehefrau verletzt worden. Herr B. hatte sich intensiv um sie gekümmert, aber zunehmend das Gefühl der Überforderung bekommen. Er beklagte Schlafstörung, Schweißausbruch, geringe Belastbarkeit, Appetit- und Gewichtsverlust, war traurig verstimmt, antriebsarm, interessenlos, gab sein Hobby auf, hatte Angst vorm Autofahren, befürchtete, nicht mehr nach Hause zu finden und zu verhungern, äußerte Suizidgedanken und war überzeugt zu verarmen. Hinzu kam die Angst vor einem erneuten Psychiatrieaufenthalt.

Am Tattag habe er plötzlich das Gefühl gehabt, jetzt handeln zu müssen. Er befürchtete, dass seine Ehefrau und er sterben müssen, und empfand ein Gefühl der Ausweglosigkeit. Herr B erstach seine Frau mit einem Küchenmesser im Wohnzimmer und versuchte, sich danach zu suizidieren. Da die Schnittverletzungen nicht zum Tod führten, versuchte er, sich in der Badewanne zu ertränken. Später rief er die Polizei, die ihn neben der Leiche seiner Frau fand.

Laut Gutachten lag eine mindestens mittlere, eher schwere depressive Episode mit psychotischen (wahnhaften) Symptomen vor. Wahnhafte Gedanken und Überzeugungen sowie die Einengung des Denkens auf belastende Inhalte führten zur Überzeugung, ,etwas tun zu müssen"; handlungsbestimmend seien wahnhafte Gedanken gewesen, nicht die depressive Verfassung. Die Steuerungsfähigkeit sei wegen „krankhafter seelischer Störung“" erheblich vermindert gewesen.

Die Gefährlichkeitsprognose sei negativ, da die Störung überdauernd sei, in künftigen Belastungssituationen sehr wahrscheinlich vergleichbar heftige Reaktionen zu erwarten seien und durch die Vorbehandlung nur eine Besserung, aber keine Symptomfreiheit erreicht werden könnte.

Das Landgericht verurteilte Herrn B. zu einer 7-jährigen Freiheitsstrafe und ordnete die Unterbringung im Maßregelvollzug nach
$\S 63$ StGB an. Im Maßregelvollzug kam es bald zur Normalisierung der Stimmung, und es trat die passiv-aggressive Persönlichkeitsstruktur mit stark rigiden Eigenschaften hervor. Auch nach Absetzen der Antidepressiva waren über mehrere Monate keine Verschlechterung von Stimmung oder Antrieb und keine wahnhaften Symptome festzustellen. Die therapeutische Mitarbeit des Patienten war sehr gering; bei Angabe von Amnesie für das Tatgeschehen war eine Deliktbearbeitung nicht möglich.

Fallbericht 2 Herr S., zum Tatzeitpunkt 48 Jahre alt, verheiratet, 2 Kinder, war Aushilfstischler in einem Bestattungsunternehmen und später dort die „rechte Hand“ des Geschäftsleiters, mit dem er seit 30 Jahren auch privat befreundet war; beide waren im Schützenverein aktiv.

Nach der Erkrankung und dem Tod der Schwiegermutter im Jahr vor der Tat kam es zu Lust- und Interessenlosigkeit sowie vermehrtem Grübeln. Geschildert wurden Antriebsstörung, Angst zu versagen, die Vorstellung, entlassen zu werden, die wahnhafte Vorstellung, der Betrieb stehe kurz vor dem Konkurs. Eine ambulante Behandlung wurde nicht konsequent betrieben; antidepressive Medikamente wurden nur kurzfristig und unregelmäßig eingenommen.

Im näheren Tatvorfeld seien quälende Suizidgedanken, seine Familie und sich selbst zu erschießen, aufgetreten, die sich auf den Chef und dessen Lebensgefährtin ausdehnten. Vor der Tat sei ihm keine Distanzierung von Suizid- und Tötungsgedanken mehr möglich gewesen.

Am Tattag holte Herr S. seine Pistole von Zuhause, erschoss im Betrieb wortlos den Chef und dessen Lebensgefährtin, rief dann Polizei und Ehefrau an und wartete auf die Festnahme. Wut und Hassgefühle wurden verneint; er habe sich wie einen Automaten erlebt, wie in einem Schockzustand. Der Selbsttötungsimpuls konnte nach der Tat nicht umgesetzt werden, dazu fehlten Herrn S. Kraft und Mut. Erinnerungsunschärfen bestanden für das Tatgeschehen.

Laut Gutachtendiagnose bestand eine schwere depressive Episode mit psychotischen Symptomen, die zur unvollständigen erweiterten Selbsttötungshandlung führte.

Das Landgericht G. erkannte auf Schuldunfähigkeit (§ $20 \mathrm{StGB})$ wegen „krankhafter seelischer Störung“ und ordnete die Unterbringung gemäß $\S 63 \mathrm{StGB}$ an. Eine Aussetzung des Vollzugs sei nicht möglich, da die ambulante Behandlung unzureichend war und während der einstweiligen Unterbringung eine wesentliche und anhaltende Befundverschlechterung eintrat.

Herr S. war in den ersten Jahren der Unterbringung wahnhaft überzeugt, er werde abgeholt und hingerichtet. Zum Jahrestag der Tat kam es wiederholt zu einer Befundverschlechterung, die eine Erhöhung der Neuroleptikadosis erforderte. Im Jahr 2005 ließen die Ängste nach und die Denkstörungen waren rückläufig, 2006 begann die Suche nach einer nachsorgenden Einrichtung, 2008 das Probewohnen in einer stationären Wohngruppe und 2009 befürwortete ein externes Prognosegutachten die Aussetzung der Unterbringung. 


\section{Erweiterter Suizid}

Tötungshandlungen wahnhaft Depressiver sind in einigen Fällen auch als erweiterte Suizide intendiert. Bei erweitertem Suizid werden andere, meist nahestehende Personen, ohne deren Wissen und Einverständnis in die suizidalen Handlungen einbezogen. Davon abzugrenzen sind die auf gemeinsamem Todeswunsch beruhenden und nach gemeinsamer Planung durchgeführten Sonderformen (Doppelsuizid, Familiensuizid) sowie die mit primär egoistischen Zielen durchgeführten Tötungshandlungen.

Erweiterte Suizide oder Mitnahmesuizide machen laut Böker u. Häfner [9] knapp 14 \% aller Gewaltverbrechen aus, die von psychisch Kranken begangen werden. Die Inzidenz liegt nach verschiedenen Untersuchungen bei $0,2-0,3$ Vorfällen/100.000 Einwohner und Jahr [10]. In den USA sind jährlich $1,5 \%$ der Suizide und $5 \%$ der Homizide erweiterte Suizide [11]. Laut Malphurs u. Cohen [12] sind $3 \%$ der männlichen erwachsenen, $11 \%$ der weiblichen erwachsenen und $19 \%$ der kindlichen Opfer von Tötungsdelikten Opfer erweiterter Suizidhandlung.

Entscheidendes Kriterium des erweiterten Suizids ist die zentrale Position des Suizids, wobei das primäre Handeln des Täters auf den Suizid ausgerichtet war, aus dem sekundär dann die Tötung der Mitnahmeopfer erwuchs [13]. Oft fehlt nach der Tötung der Mitnahmeopfer - häufig handelt es sich um nahe Angehörige, in den meisten Fällen Kinder - die notwendige Energie für einen erfolgreichen Suizid, sodass die Täter überleben. Gerade in solchen Fällen sind die sorgfältige Befunderhebung und Diagnostik von besonderer Bedeutung, bei der der psychiatrische Sachverständige sehr genau darauf zu achten haben wird, dass nicht der Begriff des erweiterten Suizids als Erklärung für ein Geschehen benutzt wird, das u. U. besser als Suizidversuch des Täters nach einem Tötungsdelikt beschrieben wird.

Der erweiterte Suizid wird oft als typisches Gewaltdelikt in der Depression angesehen. Von 10 „Homocide-suicide“Tätern einer finnischen Studie, die fast 1400 Suizide analysierte, hatten jedoch nur 3 eine Major Depression [14]. Dagegen litten 6 der 9 von Meszaros u. Fischer-Danzinger [15] untersuchten Frauen, die einen ernsthaften erweiterten Suizid begangen hatten, unter einer psychotischen Depression. Foerster [16] beschreibt einige Probleme, die mit dem Begriff ,erweiterter Suizid“"verbunden sind, und schlägt vor, entsprechende Handlungen nach rein formalen Kriterien als Tötung mit nachfolgendem Suizid(versuch) zu deklarieren.

Dem ist entgegenzusetzen, dass zugleich mit dem Begriff „erweiterter Suizid“ auch ein für das Verständnis entsprechender Verhaltensweisen bedeutsamer psychopathologischer Kontext aufgegeben wird. Die Kritik verfängt nach Meinung der Autoren dann nicht mehr, wenn der Terminus hinreichend präzisiert werden kann. Dementsprechend ist der Begriff des erweiterten Suizids der Beschreibung von
Tötungshandlungen vorzubehalten, die ätiologisch in einer Depression wurzeln und stimmungskongruente sowie pseudoaltruistische Motive aufweisen. Motive egozentrischer Art, wie Rache, Vergeltung, Empörung und verfolgende Tötungshandlungen, werden damit nicht umfasst [17].

Kriterien des erweiterten Suizids sind:

1. Primär und zentral ist der Wille des Täters zur Selbsttötung.

2. Der Wille zum Suizid wurzelt ätiologisch in der depressiven Erkrankung/Störung des Täters.

3. Mindestens eine zweite Person wird in die Suizidhandlung ohne deren Einverständnis einbezogen.

4. Zusammentreffen der Handlungen, ohne dass der Suizid eine Konsequenz des Homizids ist.

5. Ein stimmungskongruentes, pseudoaltruistisches Motiv für die Tötung des Opfers/der Opfer, keine egozentrischen Motive (Rache, Vergeltung, Empörung).

Der Begriff „homicide suicide“ leistet diese Differenzierung nicht, da er bei fehlendem Bezug zur Ätiologie einer zugrunde liegenden Störung sowohl Taten mit suizidaler als auch Taten mit kriminologischer Motivation bezeichnet.

\section{Forensisch-psychiatrische Beurteilung}

Die psychiatrische Begutachtung wahndeterminierter Tötungshandlungen hebt auf die Pathogenese der Tat ab. Die Begutachtung hat sich auf die psychopathologische Symptomatik zu stützen und bei erweiterten Suiziden wohl noch stärker als bei Homiciden die Trennung der Ebene der Befunderhebung von der Ebene der Interpretation und Wertung zu beachten [18].

Die Annahme eines erweiterten Suizids impliziert eine schwere Depression als dem Tatgeschehen zugrunde liegende psychische Störung. Aber, auch wenn die Symptome einer schweren Depression unzweifelhaft vorliegen, ist zu bedenken, dass nicht die diagnostischen Kategorien für die Begutachtung der Schuldfähigkeit entscheidend sind, sondern die psychopathologischen Auffälligkeiten im Tatvorfeld und beim Tatgeschehen. Anhand entsprechender Auffälligkeiten und Veränderungen ist zwischen prä- und postdeliktischer Depression zu unterscheiden und zu beurteilen, ob die bei der Begutachtung diagnostizierte Depression Tatanlass oder Reaktion auf die Tat ist.

Dementsprechend problematisch ist die Beschreibung der inkriminierten Handlung nach bloßen formalen Kriterien als Tötungshandlung mit nachfolgendem Suizid(versuch). Handelt es sich tatsächlich um einen erweiterten Suizid, so kann dessen planvolle Vorbereitung oder sogar ein dissimulierendes Verhalten im Vorfeld der Tat, um die Suizidabsicht auch wirklich realisieren zu können, über die Schwere der depressiven Störung täuschen und auf eine Steuerungsfähigkeit erkannt werden, die real nicht mehr vorhanden war. 
Die Erhebung des psychopathologischen Befundes kann bei der Motivationsanalyse die Frage beantworten helfen, ob von einem wahnhaft motivierten Geschehen auszugehen oder ob das Motiv für die Tötungshandlung beispielsweise Trennungsangst gewesen ist.

Bei wahnhafter Depression ist Schuldunfähigkeit gemäß $\S 20$ StGB wegen Aufhebung der Einsichts- bzw. Steuerungsfähigkeit möglich. Dabei kann allein die affektive Veränderung so ausgeprägt sein, dass damit bereits die Eingangsvoraussetzung des $\S 20$ StGB erreicht wird. Bei geringerer Schwere der psychopathologischen Symptomatik ist ggf. „nur“ verminderte Schuldfähigkeit gemäß $§ 21$ StGB anzunehmen.

\section{Unterbringung in einem psychiatrischen Krankenhaus nach § 63 StGB}

Hat jemand eine rechtswidrige Tat im Zustand der Schuldunfähigkeit (§ $20 \mathrm{StGB})$ oder der verminderten Schuldfähigkeit (§ $21 \mathrm{StGB}$ ) begangen, so ordnet das Gericht die Unterbringung in einem psychiatrischen Krankenhaus an, wenn die Gesamtwürdigung des Täters und seiner Tat ergibt, dass von ihm infolge seines Zustands erhebliche rechtswidrige Taten zu erwarten sind und er deshalb für die Allgemeinheit gefährlich ist.

Die Unterbringung gemäß $\S 63 \mathrm{StGB}$ fordert neben der Diagnose eine prognostische Einschätzung hinsichtlich der Auswirkungen der diagnostizierten Störung. Die Gefährlichkeitsprognose hat sich an Verlauf und Behandelbarkeit der Depression zu orientieren sowie u. a. Phasenhäufigkeit, Vorbehandlungen und etwaiges Auftreten von Prodromalsymptomen einzubeziehen. Allgemein gelten Depressionen als therapeutisch gut beeinflussbar; die psychopharmakologische Behandlung ermöglicht in vielen Fällen nach Beendigung der depressiven Phase eine ambulante Behandlung. Eine bis zur Hauptverhandlung anhaltende psychopathologische Symptomatik mit Suiziddynamik kann die Unterbringung gemäß $\S 63$ StGB erforderlich machen.

Wahnhafte Symptome in einer depressiven Episode haben therapeutische und kurzzeitprognostische Implikationen, denn die Behandlung der wahnhaften Depression ist ein bisher nicht befriedigend gelöstes Problem der Psychopharmakologie. Psychotisch Depressive sind schwieriger zu behandeln als nichtpsychotisch Depressive. Sie erhalten häufiger Neuroleptika, und die Rückbildung der Symptome dauert signifikant länger [8]. Bei schweren wahnhaften und therapieresistenten Depressionen wird vermehrt mit Elektrokrampftherapie behandelt [19]. Neueren Studien zufolge ist die Monotherapie mit Trimipramin der Kombination von Antidepressiva und Neuroleptika ebenbürtig [20]. Die Wirksamkeit der Kombination Pharmakotherapie mit Psy- chotherapie ist bei wahnhafter Depression nicht eindeutig belegt [21].

\section{Fazit}

Wahnhaft motivierte Tötungsdelikte schwer depressiver Patienten bedeuten eine besondere Herausforderung für den forensischen Sachverständigen hinsichtlich der Begutachtung. Die Begutachtung der Schuldfähigkeit stützt sich auf die Erhebung der psychopathologischen Symptome, die hinsichtlich ihrer Art und Schwere sowie ihres Einflusses auf motivationale Aspekte beurteilt wird. Auch bei der Beurteilung der Fälle von Tötungshandlungen wahnhaft Depressiver mit folgendem Suizidversuch ist die Erhebung des psychopathologischen Befundes die Basis der Beurteilung. Der Begriff „erweiterter Suizid“ beschreibt Tötungshandlungen, die ätiopathogenetisch in einer Depression wurzeln und stimmungskongruente sowie pseudoaltruistische Motive aufweisen.

Die Gefährlichkeitsprognose hat den Verlauf und die Erkenntnisse über die Behandelbarkeit der Depression einzubeziehen. Im Allgemeinen sind Depressionen relativ gut behandelbar, und eine länger dauernde stationäre Unterbringung ist nicht erforderlich. Bei anhaltender psychopathologischer Symptomatik mit Suiziddynamik wird die Unterbringung gemäß $\S 63$ StGB nach einem schwerwiegenden Delikt nicht zu umgehen sein.

In kontrollierten Studien sollten weitere Informationen über die Wirksamkeit einer Monotherapie mit Trimipramin bei wahnhaft depressiven Maßregelpatienten gesammelt werden. Es fehlen außerdem belastbare Daten, die einen Vergleich ermöglichen zwischen Patienten, die nach einem erweiterten Suizidversuch im Maßregelvollzug nach $\S 63 \mathrm{StGB}$ waren, und denjenigen, bei denen eine derartige Maßnahme nicht angeordnet bzw. vollzogen wurde. Es fehlt auch an gesichertem Wissen über die relevanten Kriterien für die Prognose des Rückfallrisikos bei erweitertem Suizid.

Interessenkonflikt Es bestehen keine Interessenkonflikte.

Open Access Dieser Artikel unterliegt den Bedingungen der Creative Commons Attribution Noncommercial License. Dadurch sind die nichtkommerzielle Nutzung, Verteilung und Reproduktion erlaubt, sofern der/die Originalautor/en und die Quelle angegeben sind.

\section{Literatur}

1. Wittchen HU, Jacobi F (2006) Epidemiologie der Depression. In: Stoppe G, Bramesfeld A, Schwartz FW (Hrsg) Volkskrankheit Depression. Bestandsaufnahme und Perspektiven. Springer, Berlin Heidelberg New York Tokio, S 15-37 
2. Leygraf N (1988) Psychisch kranke Straftäter. Springer, Berlin Heidelberg New York Tokio

3. Seifert D, Leygraf N (1997) Die Entwicklung des psychiatrischen Maßregelvollzugs ( $\$ 63$ StGB) in Nordrhein-Westfalen. Psychiatr Prax 24:237-244

4. Tellenbach H (1983) Melancholie. Problemgeschichte, Endogenität, Typologie. Pathogenese, Klinik. Springer, Berlin Heidelberg New York Tokio

5. Tölle R (1998) Wahn bei Depression. Nervenarzt 69:956-960

6. Venzlaff U, Schmidt-Degenhard M (2004) Affektive Psychosen. In: Foerster K (Hrsg) Psychiatrische Begutachtung. Urban \& Fischer, München, S 155-166

7. Wolfersdorf M, Frank U, Jokusch U et al (1997) Der erweiterte Suizidversuch bei depressiven Patienten und seine Folgen. Psychiatr Prax 24:270-274

8. Maj M, Pirozzi R, Magliano L et al (2007) Phenomenology and prognostic significance of delusion in major depressive disorder: a 10-year prospective follow-up-study. J Clin Psychiatry 68:1411-1417

9. Böker W, Häfner H (1973) Gewalttaten Geistesgestörter. Springer, Berlin Heidelberg New York

10. Cohen D (2002) Estimating the incidence of homicide-suicide in the United States. J Ment Health Aging 8(3):179-181

11. Marzuk M, Tardiff K, Hirsch CS (1992) The epidemiology of murder-suicide. JAMA 267:3179-3183

12. Malphurs JE, Cohen D (2002) A newspaper surveillance study of homicide-suicide in the United States. Am J Forensic Med Pathol 23(2):142-148
13. Haller R (1996) Das psychiatrische Gutachten. Manz, Wien

14. Saleva O, Putkonen H, Kiviruusu O, Lönnqvist J (2007) Homicide-suicide - An event hard to prevent and separate from homicide or suicide. Forensic Sci Int 166:204-208

15. Meszaros K, Fischer-Danzinger D (2000) Extended suicide attempt: psychopathology, personality and risk factors. Psychopathology 33:5-10

16. Foerster K (2009) „Erweiteter Suizid“. Ein problematischer Begriff? Nervenarzt 80:1078-1084

17. Okumura Y, Kraus A (1996) Zwölf Patientinnen mit erweiterter Selbsttötung: Psychologie, Persönlichkeit, Motivation, Vorgeschichte und psychosoziale Situation. Fortschr Neurol Psychiatr 64:184-191

18. Cording C, Saß H (2009) Begutachtung der ,freien Willensbestimmung" bei Suizid in der Lebensversicherung. Nervenarzt 80:1070-1077

19. Deutsche Gesellschaft für Psychiatrie, Psychotherapie und Nervenheilkunde (Hrsg) (2000) Behandlungsleitlinie Affektive Erkrankungen, Redaktion: W. Gaebel, P. Falkai. Steinkopff, Berlin

20. Künzel HE, Ackl A, Hatzinger M et al (2009) Outcome in delusional depression comparing trimipramine monotherapy with a combination of amitriptyline and haloperidol - a double-blind multicenter trial. J Psychiatr Res 43:702-710

21. Gaudiano BA, Beevers CG, Miller IW (2005) Differential response to combined treatment in patients with psychotic versus nonpsychotic major depression. J Nerv Ment Dis 193:625-628 